PROCEEDINGS OF THE

AMERICAN MATHEMATICAL SOCIETY

Volume 130, Number 4, Pages 1035-1042

S 0002-9939(01)06383-3

Article electronically published on October 1, 2001

\title{
RIGIDITY OF PROPER HOLOMORPHIC MAPPINGS BETWEEN EQUIDIMENSIONAL BOUNDED SYMMETRIC DOMAINS
}

\author{
ZHEN-HAN TU
}

(Communicated by Steven R. Bell)

\begin{abstract}
We prove that any proper holomorphic mapping between two equidimensional irreducible bounded symmetric domains with rank $\geq 2$ is a biholomorphism. The proof of the main result in this paper will be achieved by a differential-geometric study of a special class of complex geodesic curves on the bounded symmetric domains with respect to their Bergman metrics.
\end{abstract}

\section{INTRODUCTION}

In 1977, Alexander [1] proved the following fundamental result.

Theorem 1.A (Alexander [1]). Any proper holomorphic mapping of the unit ball in $\mathbb{C}^{n}(n \geq 2)$ into itself is an automorphism.

Alexander's theorem has been generalized to several classes of domains. Especially, there are many important results concerning proper holomorphic mappings between bounded pseudoconvex domains in $\mathbb{C}^{n}$ with smooth boundary, and these researches are often heavily based on analytic techniques (e.g., see survey articles of Bell and Narasimhan [5] and Forstnerič [7]). In this topic, respectively, Bedford and Bell [2] and Diederich and Fornæss [6] proved the following results.

Theorem 1.B (Bedford and Bell [2]). Let D be a bounded weakly pseudoconvex domain in $\mathbb{C}^{n}(n \geq 2)$ with smooth real-analytic boundary. Then any proper holomorphic self-mapping of $D$ is an automorphism.

Theorem 1.C (Diederich and Fornæss [6). If $\Omega, D \subset \mathbb{C}^{n}(n \geq 2)$ are smoothly bounded pseudoconvex domains and $\Omega$ is strongly pseudoconvex, then any proper holomorphic mapping of $\Omega$ onto $D$ is a local biholomorphism. Thus, if $D$ is simply connected, then the mapping is biholomorphic.

Even though the bounded homogeneous domains in $\mathbb{C}^{n}$ are always pseudoconvex, there are, of course, many such domains (e.g., all bounded symmetric domains of rank $\geq 2$ ) without smooth boundary. The lack of boundary regularity usually presents a serious analytical difficulty. In 1984, by using results of Bell [3] and

Received by the editors September 29, 2000.

2000 Mathematics Subject Classification. Primary 32H02, 32M15.

Key words and phrases. Bounded symmetric domains, Hermitian symmetric manifolds, proper holomorphic mappings, rigidity, totally geodesic submanifolds.

(C)2001 American Mathematical Society 
Tumanov-Henkin [20, Henkin and Novikov 9] proved the following result (see Theorem 3.3 in Forstnerič [7] for references).

Theorem 1.D (Henkin and Novikov [9]). Any proper holomorphic self-mapping on an irreducible bounded symmetric domain of rank $\geq 2$ is an automorphism.

The subject of Hermitian symmetric manifolds plays a central role in the domain of complex differential geometry. Of particular importance is the phenomenon of rigidity of complex structures of such manifolds (e.g., see Siu [15, 16]). In 1987, Mok [10, 11] obtained metric rigidity theorems on Hermitian locally symmetric manifolds, and their proofs are applied to the study of holomorphic mappings between Hermitian locally symmetric manifolds of the same type, yielding various rigidity theorems on holomorphic mappings (see Mok [12]). Inspired by the idea in Mok's work [10, 11, Tsai [17, 18, obtained some interesting results on this topic. Following this line, we shall prove the following.

Theorem 1.1. Let $\Omega_{1}$ and $\Omega_{2}$ be two equidimensional bounded symmetric domains. Assume that $\Omega_{1}$ is irreducible and $\operatorname{rank}\left(\Omega_{1}\right) \geq 2$. Then, any proper holomorphic mapping $f$ from $\Omega_{1}$ to $\Omega_{2}$ is a biholomorphism.

In Theorem 1.1, the assumption " $\Omega_{1}$ is irreducible" cannot be removed. For example, $f\left(z_{1}, \ldots, z_{n}\right)=\left(z_{1}^{2}, \ldots, z_{n}^{2}\right)$ is a proper holomorphic mapping from the polydisc $D^{n} \subset \mathbb{C}^{n}$ into itself but $f$ is not a biholomorphism. If the assumption "rank $\left(\Omega_{1}\right) \geq 2 "$ in Theorem 1.1 is removed, then, by Bell [4] and Theorem 1.A, Theorem 1.1 holds when $\operatorname{dim}\left(\Omega_{1}\right) \geq 2$.

We shall now present an outline of the argument in our proof of Theorem 1.1. Let $D$ be a bounded symmetric domain with Bergman metric. The minimal discs on $D$ are a special class of complex geodesic curves on $D$ such that their unit holomorphic tangent vectors realize the minimum of the holomorphic sectional curvature of $D$. The proof of Theorem 1.1 will be achieved by a differential-geometric study of the minimal discs on $\Omega_{1}$ and $\Omega_{2}$. In order to prove that $f: \Omega_{1} \rightarrow \Omega_{2}$ is a biholomorphism, the key step in our proof is to verify that $f$ maps all minimal discs on $\Omega_{1}$ to minimal discs on $\Omega_{2}$. We finish this step by using Bell [4] (to conclude that $f$ is algebraic) and exploiting the characteristic symmetric submanifolds and holomorphic boundary components of two equidimensional bounded symmetric domains. Then, an elementary proof of Theorem 1.1 can be given.

\section{BACKGROUND AND PRELIMINARIES}

In this section we collect some basic facts about Hermitian symmetric manifolds, including some results of Mok and Tsai [13, and refer to Mok [12] and Wolf [21] for further details.

Let $X_{o}$ be an irreducible Hermitian symmetric space of non-compact type. Fix a point $o \in X_{o}$. Write $X_{o}=G_{o} / K$ as usual, where $G_{o}$ is the identity component of the isometry group of $X_{o}$ and $K$ is the isotropy subgroup at $o$. The Lie algebra $\mathfrak{g}_{o}$ of $G_{o}$ has a Cartan decomposition $\mathfrak{g}_{o}=\mathfrak{l}+\mathfrak{m}_{o}$ with respect to the symmetry at $o$. Complexifying $\mathfrak{m}_{o}$ gives $\mathfrak{m}=\mathrm{m}_{o}^{\mathbb{C}} \cong \mathfrak{m}^{+} \oplus \mathfrak{m}^{-}$so that $\mathfrak{m}^{+}$is identified with $T_{o}\left(X_{o}\right)$, the holomorphic tangent space at $o$. Denote by $G$ and $\mathfrak{g}$ the complexified Lie group and Lie algebra of $G_{o}$ and $\mathfrak{g}_{o}$ respectively. Write $X_{c}=G / P$ for the compact dual of $X_{o}$ as usual, where $P$ is the parabolic subgroup of $G$ corresponding to the parabolic subalgebra $\mathfrak{l}^{\mathbb{C}} \oplus \mathfrak{m}^{-}$. The map $g K \rightarrow g P$ from $G_{o} / K$ to $G / P$ embeds $X_{o}$ holomorphically as an open subset of $X_{c}$. It is called the Borel embedding. Let 
exp denote the exponential map from $\mathfrak{m}^{+}$to $G$. The Harish-Chandra embedding theorem tells us that the map $\tau: \mathfrak{m}^{+} \rightarrow X_{c}$ given by $\tau(m)=\exp (m) P$ is a complex analytic diffeomorphism of $\mathfrak{m}^{+}$onto a dense open subset of $X_{c}$ that contains $X_{o}$ and $\tau^{-1}\left(X_{o}\right)$ is a bounded symmetric domain in $\mathfrak{m}^{+}$. Here and henceforth we identify $\mathfrak{m}^{+}$with $\mathbb{C}^{N}$ and the Euclidean coordinates on $\mathbb{C}^{N}$ are called HarishChandra coordinates.

Let $\mathfrak{h}$ be a Cartan subalgebra of $\mathfrak{l}$. Then $\mathfrak{h}^{\mathbb{C}}$ is also a Cartan subalgebra of $\mathfrak{g}$. Denote by $\Delta$ the $\mathfrak{h}^{\mathbb{C}}$-root system of $\mathfrak{g}$. Two roots are called strongly orthogonal if and only if $\phi \pm \varphi \notin \Delta$. We construct a maximal strongly orthogonal set $\left\{\phi_{1}, \ldots, \phi_{r}\right\}$ $\left(r=\right.$ rank of $\left.X_{o}\right)$, denoted by $\Pi$, of noncompact positive roots according to HarishChandra. Let $\Lambda$ be a proper subset of $\Pi$. Denote by $\mathfrak{g}_{\Lambda}$ the derived algebra of $\mathfrak{h}^{\mathbb{C}}+\sum_{\phi \perp \Pi \backslash \Lambda} \mathfrak{g}^{\phi}$. Let $G_{\Lambda}$ be the Lie subgroup of $G$ for $\mathfrak{g}_{\Lambda}$ and define $G_{\Lambda, o}=G_{\Lambda} \cap G_{o}$. Let $X_{\Lambda}=G_{\Lambda}(o) \subset X_{c}$ and $X_{\Lambda, o}=G_{\Lambda, o}(o) \subset X_{o}$. With these notations we have:

Proposition 2.A (Wolf [21]). $X_{\Lambda}$ and $X_{\Lambda, o}$ are totally geodesic Hermitian symmetric subspaces of $X_{c}$ and $X_{o}$ respectively with rank $|\Lambda|$. If $\left|\Lambda_{1}\right|=\left|\Lambda_{2}\right|$, where $|\Lambda|$ denotes the number of elements in it, then $X_{\Lambda_{1}, o}$ is isomorphic to $X_{\Lambda_{2}, o}$ via a holomorphic isometry of $X_{o}$.

$X_{\Lambda, o}$ (resp. $\left.X_{\Lambda}\right)$ are called the characteristic symmetric subspaces of $X_{o}$ (resp. $\left.X_{c}\right)$. In particular, $X_{\Lambda, o}$ (resp. $X_{\Lambda}$ ) with $|\Lambda|=|\Pi|-1$ are called the maximal characteristic symmetric subspaces of $X_{o}$ (resp. $X_{c}$ ) and all intermediate characteristic symmetric subspaces of $X_{o}$ (resp. $X_{c}$ ) can be obtained from the maximal characteristic symmetric subspaces of $X_{o}$ (resp. $X_{c}$ ) inductively.

We now define the boundary components of $X_{o} \subset X_{c}$. Two points of $\partial X_{o}$ are said to be in the same equivalence class if one can be connected to the other by a chain of holomorphic arcs. The equivalence classes are called the boundary components of $X_{o}$. Let's define the Cayley transformation $c_{\Pi \backslash \Lambda} \in G$ to be

$$
c_{\Pi \backslash \Lambda}:=\exp \left(\frac{\pi}{4} \sum_{\phi \in \Pi \backslash \Lambda} e_{-\phi}-e_{\phi}\right) .
$$

Then we have

Proposition 2.B (Wolf [21]). The boundary components of $X_{o} \subset X_{c}$ are just the sets $k \cdot c_{\Pi \backslash \Lambda} \cdot X_{\Lambda, o}$ with $k \in K$ and $\Lambda \subset \Pi$. They are Hermitian symmetric manifolds of the noncompact type with rank $|\Lambda|$. The action of $G_{o} \subset G$ is transitive on the union of those boundary components of the same rank. The topological boundary of $X_{O}$ is therefore the disjoint union of boundary orbits under the action of $G_{O}$.

Thus the maximal boundary components and the maximal characteristic symmetric subspaces of a bounded symmetric domain must have the same dimension. Now we describe the boundary components explicitly.

Proposition 2.C (Wolf [21]). Let $X_{o} \subset X_{c}$ be the Borel embedding of an irreducible Hermitian symmetric manifold of the noncompact type with rank $r$. For each integer $m, 0 \leq m<r$, there is just one $G_{o}$-equivalence class $\left\{X_{m, o}\right\}$ of boundary component with rank $m$ for $X_{o} \subset X_{c} . X_{0, o}$ is a single point and $X_{m, o}$ are given as follows with $X_{r, o}=X_{o}$ :

I. For $X_{o}=D_{p, q}^{I}(r=p \leq q), X_{m, o}=D_{m, q-p+m}^{I}$.

II. For $X_{o}=D_{2 r}^{I I}, X_{m, o}=D_{2 m}^{I I}$ and for $X_{o}=D_{2 r+1}^{I I}, X_{m, o}=D_{2 m+1}^{I I}$.

III. For $X_{o}=D_{r}^{I I I}, X_{m, o}=D_{m}^{I I I}$. 
IV. For $X_{o}=D_{n}^{I V}$ (here $r=2$ ), $X_{1, o}$ is the unit disc in $\mathbb{C}^{1}$.

V. For $X_{o}=D^{V}$ (here $r=2$ ), $X_{1, o}$ is the unit ball in $\mathbb{C}^{5}$.

VI. For $X_{o}=D^{V I}$, (here $r=3$ ), $X_{2, o}=D_{10}^{I V}$ and $X_{1, o}$ is the unit disc in $\mathbb{C}^{1}$.

For a proof of Proposition 2.C, see pg. 292 of Wolf 21. A very elementary proof by function theory technique for (I) of Proposition 2.C is given in pp. 93-95 of Pyatetskii-Shapiro [14]. Since characteristic symmetric submanifolds are isomorphic to boundary components via $k \cdot c_{\Pi \backslash \Lambda}$ with $k \in K$ and $\Lambda \subset \Pi$, this explicitly describes characteristic symmetric submanifolds as well.

The minimal rational curves are precisely those totally geodesic cuves on $X_{c}$ with respect to any possible choice of canonical (Kähler-Einstein) metrics on $X_{c}$. Via the Harish-Chandra embedding $X_{o} \cong \Omega \subset \subset \mathbb{C}^{N} \subset X_{c}$ we call the intersections of $C$ with $\mathbb{C}^{N}$ (resp. $\Omega$ ) minimal affine lines (resp. minimal discs). The characteristic vectors of $X_{o}$ (resp. $X_{c}$ ) are defined as those nonzero vectors tangent to minimal discs $D=C \cap \Omega$ (resp. minimal rational curves $C$ ). Then the unit characteristic vectors in $T_{p}\left(X_{o}\right)$ (resp. $T_{p}\left(X_{c}\right)$ ) are exactly those unit vectors which realize the minimum (resp. maximum) of the holomorphic sectional curvature of $X_{o}$ (resp. $X_{c}$ ) with the canonical metric at $p$. Let $X$ denote $X_{o}$ or $X_{c}$. Denote by $\tilde{\mathcal{S}}_{p}(X) \subset$ $T_{p}(X)$ the cone of all characteristic vectors of $X$ at $p$, and the characteristic variety $\mathcal{S}_{p}(X) \subset \mathbb{P} T_{p}(X)$ is defined as the projectivization of $\tilde{\mathcal{S}}_{p}(X)-\{0\}$, which is a connected complex submanifold of $\mathbb{P} T_{p}(X)$. For an irreducible bounded symmetric domain $D$ (in their standard realizations) in $\mathbb{C}^{n}$, define $\mathcal{S}(D):=\bigcup_{p \in D} \mathcal{S}_{p}(D) \subset$ $\mathbb{P} T(D)$. If we identify $\mathbb{P} T(D)$ with $D \times \mathbb{P}^{n-1}$ by using the Euclidean coordinates, then $\mathcal{S}(D) \cong D \times \mathcal{S}_{o}(D)$, i.e., $\mathcal{S}(D)$ is parallel on $D$ in the Euclidean sense (see Proposition 2 on pg. 103 of Mok [12]). The characteristic varieties $\mathcal{S}_{p}(X) \subset \mathbb{P} T_{p}(X)$ are given explicitly corresponding to 6 types of irreducible bounded symmetric domains $X$ on pg. 249 of Mok 12. Fix a characteristic vector $\alpha \in T_{o}(X)\left(X=X_{c}\right.$ or $X_{o}$ ); by Mok [12] we have an orthogonal decomposition $T_{o}(X)=\mathbb{C} \alpha \oplus \mathcal{H}_{\alpha} \oplus \mathcal{N}_{\alpha}$ into eigenspaces of the Hermitian form $Q_{\alpha}(\xi, \eta)=R_{\alpha \bar{\alpha} \xi \bar{\eta}}$ corresponding to eigenvalues $R_{\alpha \bar{\alpha} \alpha \bar{\alpha}}, \frac{1}{2} R_{\alpha \bar{\alpha} \alpha \bar{\alpha}}$ and 0 . Then we have $\mathcal{N}_{\alpha}=T_{o}\left(X_{\Lambda, o}\right)$ for $\Lambda=\Pi \backslash \alpha$ by Mok and Tsai [13]. Let $n(X)=\operatorname{dim}_{\mathbb{C}} X_{\Lambda, o}=\operatorname{dim}_{\mathbb{C}} \mathcal{N}_{\alpha}$. Then $\operatorname{dim}_{\mathbb{C}} \mathcal{N}_{\alpha}$ is maximum among non-zero vectors $\mu \in T_{o}(X)$ and we call $n(X)$ the null dimension of $X$.

Like Tsai [18, our idea in this paper is based on the following two propositions.

Proposition 2.1 (Tsai [18]). Let $\Omega_{1}$ and $\Omega_{2}$ be two bounded symmetric domains and let $f: \Omega_{1} \rightarrow \Omega_{2}$ be a proper holomorphic mapping. Suppose that $\Omega_{1}$ is irreducible and rank $\left(\Omega_{1}\right) \geq 2$. Then we have the following result:

(i) For any characteristic symmetric subspace $\Omega_{\Lambda}$ of $\Omega_{1}, f\left(\Omega_{\Lambda}\right)$ is contained in some characteristic symmetric subspace of $\Omega_{2}$.

(ii) $\operatorname{rank}\left(\Omega_{1}\right) \leq \operatorname{rank}\left(\Omega_{2}\right)$.

(iii) If $\operatorname{rank}\left(\Omega_{1}\right)=\operatorname{rank}\left(\Omega_{2}\right)$, then $d f\left(\tilde{\mathcal{S}}_{p}\left(\Omega_{1}\right)\right) \subset \tilde{\mathcal{S}}_{f(p)}\left(\Omega_{2}\right)$ for any $p \in \Omega_{1}$.

(iv) If $\operatorname{rank}\left(\Omega_{1}\right)=\operatorname{rank}\left(\Omega_{2}\right)$, then $\Omega_{2}$ is irreducible.

Proposition 2.2. Let $\Omega_{1}$ and $\Omega_{2}$ be two irreducible bounded symmetric domains with $\operatorname{rank}\left(\Omega_{i}\right) \geq 2(i=1,2)$ and let $f: \Omega_{1} \rightarrow \Omega_{2}$ be a proper holomorphic mapping. If $f$ maps any minimal disc of $\Omega_{1}$ into a minimal disc of $\Omega_{2}$, then $f$ is necessarily a totally geodesic isometric embedding with respect to their Bergman metrics (up to normalizing constants). 
Remark. The idea in the proof of Proposition 2.2 comes from Mok [10] (see Chapter 6 of Mok [12] for references). The proof of Proposition 2.2 in a special case is given in Tsai [18 and the proof in Tsai [18] can be easily checked to be valid in our case.

\section{Proof of Theorem 1.1}

In this section we shall give the proof of Theorem 1.1.

A domain $\Omega$ in $\mathbb{C}^{n}$ is said to be circular if and only if it is invariant under the action of the circle group $S^{1}$ given by $\left(z_{1}, \ldots, z_{n}\right) \rightarrow\left(e^{i \theta} z_{1}, \ldots, e^{i \theta} z_{n}\right)$. A circular domain is said to be complete if it contains the origin.

Proposition 3.1 (Bell [4). Suppose that $\Omega_{1}$ and $\Omega_{2}$ are bounded complete circular domains in $\mathbb{C}^{n}$. If $f: \Omega_{1} \rightarrow \Omega_{2}$ is a proper holomorphic mapping with $f(0)=0$, then $f$ must be algebraic.

Proposition 3.2. Let $\Omega_{1}$ and $\Omega_{2}$ be two equidimensional bounded symmetric domains in $\mathbb{C}^{n}$ and let $f: \Omega_{1} \rightarrow \Omega_{2}$ be a proper holomorphic mapping. Suppose $\Omega_{1}$ is irreducible and $\operatorname{rank}\left(\Omega_{1}\right) \geq 2$. Then every minimal disc of $\Omega_{1}$ is mapped by $f$ onto a minimal disc of $\Omega_{2}$.

Proof. Let $\Omega_{1}{ }^{\prime}$ be a maximal characteristic symmetric subspace of $\Omega_{1}$ (note that $\operatorname{rank}\left(\Omega_{1}{ }^{\prime}\right)=\operatorname{rank}\left(\Omega_{1}\right)-1$ here). Then, by (i) of Proposition 2.1, there exists a maximal characteristic symmetric subspace $\Omega_{2}{ }^{\prime}$ of $\Omega_{2}$ such that $f\left(\Omega_{1}{ }^{\prime}\right) \subset \Omega_{2}{ }^{\prime}$. Since $f: \Omega_{1} \rightarrow \Omega_{2}$ is algebraic by Proposition 3.1, $f: \Omega_{1} \rightarrow \Omega_{2}$ extends a holomorphic mapping $\tilde{f}: \mathbb{C}^{n}-R \rightarrow \mathbb{C}^{n}$ for a nowhere dense complex analytic set $R \subset \mathbb{C}^{n}$. Since $f: \Omega_{1} \rightarrow \Omega_{2}$ is proper, we have $\tilde{f}\left(\partial \Omega_{1} \cap\left(\mathbb{C}^{n}-R\right)\right) \subset \partial \Omega_{2}$. Since the ramification locus of $\tilde{f}$ is a nowhere dense complex analytic set, there exist $p \in \partial \Omega_{1}, q=\tilde{f}(p) \in \partial \Omega_{2}$ such that $\tilde{f}: U(p) \rightarrow U(q)$ is a biholomorphism with $\tilde{f}\left(U(p) \cap \partial \Omega_{1}\right)=U(q) \cap \partial \Omega_{2}$, where $U(p), U(q)$ are two small domains in $\mathbb{C}^{n}$ with $p \in U(p)$ and $q \in U(q)$. Since the disjoint union of maximal boundary components of a bounded symmetric domain $\Omega \subset \mathbb{C}^{n}$ is dense in the topological boundary of $\Omega \subset \mathbb{C}^{n}$ by Propositions 2.B and 2.C, the maximal boundary components of $\Omega_{1} \subset \mathbb{C}^{n}$ and $\Omega_{2} \subset \mathbb{C}^{n}$ have the same dimension. Since the maximal boundary components and the maximal characteristic symmetric subspaces of a bounded symmetric domain have the same dimension by Proposition 2.B, the maximal characteristic symmetric subspaces of $\Omega_{1}$ and $\Omega_{2}$ have the same dimension also. Thus $\left.f\right|_{\Omega_{1}{ }^{\prime}}: \Omega_{1}{ }^{\prime} \rightarrow \Omega_{2}{ }^{\prime}$ is a proper holomorphic mapping with $\operatorname{dim} \Omega_{1}{ }^{\prime}=\operatorname{dim} \Omega_{2}{ }^{\prime}$ and $\operatorname{rank}\left(\Omega_{1}{ }^{\prime}\right)=\operatorname{rank}\left(\Omega_{1}\right)-1$. By induction on rank, for any characteristic symmetric subspace $B_{1}$ of $\Omega_{1}$ with $\operatorname{rank}\left(B_{1}\right)=1$, there exists a characteristic symmetric subspace $B_{2}$ of $\Omega_{2}$ such that $f\left(B_{1}\right) \subset B_{2}$ with $\operatorname{dim} B_{1}=\operatorname{dim} B_{2}$. Since $\left.f\right|_{B_{1}}: B_{1} \rightarrow B_{2}$ is an algebraic proper holomorphic mapping by Proposition 3.1, there is no complex analytic subvariety in $\partial B_{2}$, and then $B_{2}$ must be of rank 1 . This means that $B_{1}$ and $B_{2}$ are characteristic symmetric subspaces of $\Omega_{1}$ and $\Omega_{2}$, respectively, such that $\operatorname{dim} B_{1}=\operatorname{dim} B_{2}, f\left(B_{1}\right)=B_{2}$ and $\operatorname{rank}\left(B_{1}\right)=\operatorname{rank}\left(B_{2}\right)=1$.

(i) If $\operatorname{dim} B_{1}=1$, then minimal discs on $\Omega_{1}$ and $\Omega_{2}$ are characteristic symmetric subspaces of rank 1 , and then every minimal disc of $\Omega_{1}$ is mapped by $f$ onto a minimal disc of $\Omega_{2}$.

(ii) If $\operatorname{dim} B_{1} \geq 2$, then $\left.f\right|_{B_{1}}: B_{1} \rightarrow B_{2}$ is a biholomorphism by Theorem 1.A. Since all minimal discs of the unit ball $B^{n}$ in $\mathbb{C}^{n}(n \geq 2)$ are Aut $\left(B^{n}\right)$-orbits of the unit disc $D \hookrightarrow B^{n}$ and the isometric group of $\Omega_{1}$ acts transitively on the set of 
minimal discs of $\Omega_{1}$, every minimal disc of $\Omega_{1}$ is mapped by $f$ onto a minimal disc of $\Omega_{2}$.

The proof of Proposition 3.2 is completed.

Proposition 3.3. Let $\Omega_{1}$ and $\Omega_{2}$ be two equidimensional bounded symmetric domains and let $f: \Omega_{1} \rightarrow \Omega_{2}$ be a proper holomorphic mapping. Suppose $\Omega_{1}$ is irreducible and $\operatorname{rank}\left(\Omega_{1}\right) \geq 2$. Then we have $n\left(\Omega_{1}\right) \leq n\left(\Omega_{2}\right)$ and $\Omega_{2}$ is irreducible.

Proof. $n\left(\Omega_{1}\right) \leq n\left(\Omega_{2}\right)$ immediately comes from (i) of Proposition 2.1.

Now we shall prove that $\Omega_{2}$ is irreducible. Without loss of generality we assume that $\Omega_{2}=\Omega_{2}{ }^{\prime} \times \Omega_{2}{ }^{\prime \prime}$, where $\Omega_{2}{ }^{\prime}$ and $\Omega_{2}{ }^{\prime \prime}$ are irreducible. Let $R \subset \Omega_{1}$ be the ramification locus of $f$. Fix $x \in \Omega_{1}-R$. Then by Proposition 3.2 we have

$$
d f\left(\mathcal{S}_{x}\left(\Omega_{1}\right)\right) \subset \mathcal{S}_{f(x)}\left(\Omega_{2}\right)=\mathcal{S}_{f_{1}(x)}\left(\Omega_{2}{ }^{\prime}\right) \cup \mathcal{S}_{f_{2}(x)}\left(\Omega_{2}{ }^{\prime \prime}\right) \text { (a disjoint union) }
$$

where $f(x)=\left(f_{1}(x), f_{2}(x)\right) \in \Omega_{2}{ }^{\prime} \times \Omega_{2}{ }^{\prime \prime}$.

Since $\mathcal{S}_{x}\left(\Omega_{1}\right)$ is connected, by the continuity argument we have

$$
d f\left(T\left(\Omega_{1}\right)\right) \subset T\left(\Omega_{2}{ }^{\prime}\right) \quad \text { or } \quad d f\left(T\left(\Omega_{1}\right)\right) \subset T\left(\Omega_{2}{ }^{\prime \prime}\right) .
$$

This is impossible as a proper holomorphic mapping cannot decrease dimension. We get Proposition 3.3.

We can get Theorem 1.1 by Proposition 2.2 and 3.2 now. But we shall not use Proposition 2.2 to prove Theorem 1.1 and shall give a more elementary proof here.

Proof of Theorem 1.1. The proof consists of two steps:

Step $(1) \cdot \operatorname{dim}_{\mathbb{C}}\left(\mathcal{S}_{o}\left(\Omega_{1}\right)\right)=\operatorname{dim}_{\mathbb{C}}\left(\mathcal{S}_{o}\left(\Omega_{2}\right)\right)$.

Proof of Step (1). Let $R \subset \Omega_{1}$ be the ramification locus of $f$. For $x \in \Omega_{1}-R$ and $y=f(x), f$ induces an isomorphism $\sigma=[d f(x)]: \mathbb{P} T_{x}\left(\Omega_{1}\right) \rightarrow \mathbb{P} T_{y}\left(\Omega_{2}\right)$. By Proposition 3.2 we have $\sigma\left(\mathcal{S}_{x}\left(\Omega_{1}\right)\right) \subset \mathcal{S}_{y}\left(\Omega_{2}\right)$. On the other hand, Since $n\left(\Omega_{1}\right) \leq$ $n\left(\Omega_{2}\right)$ by Proposition 3.3 and $\operatorname{dim}_{\mathbb{C}}\left(\mathcal{S}_{p}(X)\right)+n(X)=\operatorname{dim}_{\mathbb{C}}(X)-1$ (where $p \in X$ and $X=X_{c}$ or $\left.X_{o}\right)$ by Prop. 4 on pg. 105 of Mok [12], we have $\operatorname{dim}_{\mathbb{C}}\left(\mathcal{S}_{x}\left(\Omega_{1}\right)\right) \geq$ $\operatorname{dim}_{\mathbb{C}}\left(\mathcal{S}_{y}\left(\Omega_{2}\right)\right)$. Since $\mathcal{S}_{y}\left(\Omega_{2}\right)$ is connected by Proposition 3.3, we have $\sigma\left(\mathcal{S}_{x}\left(\Omega_{1}\right)\right)=$ $\mathcal{S}_{y}\left(\Omega_{2}\right)$. As $\operatorname{dim}_{\mathbb{C}}\left(\mathcal{S}_{p}(X)\right)$ is independent of $p \in X$, we get Step (1).

Step (2). $f: \Omega_{1} \rightarrow \Omega_{2}$ is a biholomorphism.

Proof of Step (2). Let $J_{f}(z)$ be the Jacobian determinant of $f$ at $z \in \Omega_{1}$ and let $R_{f}=\left\{z \in \Omega_{1}: J_{f}(z)=0\right\}$ be the ramification locus. Evidently, $f$ is a local biholomorphism at any point of $\Omega_{1}-R_{f}$. If $R_{f} \neq \emptyset$, then $R_{f}$ must be an analytic set of complex codimension 1 .

Now fix a point $x \in R_{f}$ and assume that $d f(x): T_{x}\left(\Omega_{1}\right) \rightarrow T_{f(x)}\left(\Omega_{2}\right)$ satisfies $d f(x)\left(T_{x}\left(\Omega_{1}\right)\right) \neq 0$. The projective linear map $[d f(x)]: \mathbb{P} T_{x}\left(\Omega_{1}\right) \rightarrow \mathbb{P} T_{f(x)}\left(\Omega_{2}\right)$ induces a rational map $\left.[d f(x)]\right|_{\mathcal{S}_{x}\left(\Omega_{1}\right)}: \mathcal{S}_{x}\left(\Omega_{1}\right) \rightarrow \mathcal{S}_{f(x)}\left(\Omega_{2}\right)$ (by Proposition 3.2) with image lying in a hyperplane section $H$ of $\mathcal{S}_{f(x)}\left(\Omega_{2}\right), H \subset$ but $\neq \mathcal{S}_{f(x)}\left(\Omega_{2}\right)$, as $\mathcal{S}_{f(x)}\left(\Omega_{2}\right)$ is not contained in any hyperplane section of $\mathbb{P} T_{f(x)}\left(\Omega_{2}\right)$. It follows that $\left.[d f(x)]\right|_{\mathcal{S}_{x}\left(\Omega_{1}\right)}: \mathcal{S}_{x}\left(\Omega_{1}\right) \rightarrow \mathcal{S}_{f(x)}\left(\Omega_{2}\right)$ must have positive dimensional generic fibers by Step (1). In particular, there exists a holomorphic one-parameter family of minimal discs $\left\{C_{z}: z \in D\right\}$ (where $D$ is a disc of the complex plane) passing through $x$ such that each $C_{z}$ is mapped by $f$ onto the same minimal disc in $\Omega_{2}$. This is impossible, as $f^{-1}(w)$ is a finite set for any point $w \in \Omega_{2}$.

So $d f(x): T_{x}\left(\Omega_{1}\right) \rightarrow T_{f(x)}\left(\Omega_{2}\right)$ satisfies $d f(x)\left(T_{x}\left(\Omega_{1}\right)\right)=0$ for all $x \in R_{f}$. In this case, all smooth points of $R_{f}$ are ramification points of $\left.f\right|_{R_{f}}: R_{f} \rightarrow f\left(R_{f}\right)$. This is impossible, as $\left.f\right|_{R_{f}}: R_{f} \rightarrow f\left(R_{f}\right)$ is a proper holomorphic mapping. 
Hence $R_{f}=\emptyset$, so that $d f(z): T_{z}\left(\Omega_{1}\right) \rightarrow T_{f(z)}\left(\Omega_{2}\right)$ for any $z \in \Omega_{1}$ is an isomorphism. Consequently, $f$ is a holomorphic covering map. As any Hermitian symmetric manifold of non-compact type is simply connected, $f: \Omega_{1} \rightarrow \Omega_{2}$ is in fact a biholomorphism. So we have Step (2).

The proof of Theorem 1.1 is completed.

\section{ACKNOWLEDGMENTS}

This work is part of the author's Ph.D. thesis [19]. The author would like to express his hearty thanks to his advisor, Professor Ngaiming Mok, who kindly introduced this topic to him. Without Professor Mok's patient guidance, encouragement and many useful suggestions, the author would not have been able to complete this work. In addition, the author wishes to thank Professors Wing-Keung To, I-Hsun Tsai and Sai-Kee Yeung for helpful disscussions.

\section{REFERENCES}

[1] H. Alexander, Proper holomorphic mappings in $\mathbb{C}^{n}$, Indiana Univ. Math. J. 26(1977), 134146. MR 54:10685

[2] E. Bedford and S. Bell, Proper self maps of weakly pseudoconvex domains, Math. Ann. 261(1982), 47-49. MR 84c:32026

[3] S. Bell, Proper holomorphic correspondences between circular domains, Comment. Math. Helv. 57(1982), 532-538. MR 84m:32032

[4] S. Bell, Algebraic mappings of circular domains in $\mathbb{C}^{n}$, in Several Complex Variables (edited by J. Fornaess), Math Notes Vol. 38, Princeton University Press, 1993, 126-135. MR 94a:32040

[5] S. Bell and R. Narasimhan, Proper holomorphic mappings of complex spaces, in Several Complex Variables VI (Barth and Narasimhan, Eds), Encyclopaedia of Math. Sciences Vol. 69, Springer-Verlag, 1990, 1-38. MR 92m:32046

[6] K. Diederich and J.E. Fornæss, Proper holomorphic images of strictly pseudoconvex domains, Math. Ann. 259(1982), 279-286. MR 83g:32026

[7] F. Forstnerič, Proper holomorphic mappings: A survey, in Several Complex Variables (edited by J. Fornaess), Math Notes Vol. 38, Princeton University Press, 1993, 297-363. MR 94a:32042

[8] S. Helgason, Differential Geometry, Lie Groups and Symmetric Spaces, Academic Press, New York, 1978. MR 80k:53081

[9] G.M. Henkin and R. Novikov, Proper mappings of classical domains, in Linear and Complex Analysis Problem Book, Lecture Notes in Math. Vol. 1043, Springer, Berlin, 1984, 625-627.

[10] N. Mok, Uniqueness theorems of Hermitian metric of seminegative curvature on quotients of bounded symmetric domains, Ann. of Math. 125(1987), 105-152. MR 88f:32076

[11] N. Mok, Uniqueness theorem of Kähler metrics of semipositive holomorphic bisectional curvature on compact Hermitian symmetric space, Math. Ann. 276(1987), 177-204. MR 88c:53063

[12] N. Mok, Metric Rigidity Theorems on Hermitian Locally Symmetric Manifolds, Series in Pure Math. Vol. 6, World Scientific, Singapore, 1989. MR 92d:32046

[13] N. Mok and I-H. Tsai, Rigidity of convex realizations of irreducible bounded symmetric domains of rank $\geq 2$, J. Reine Angew. Math. 431(1992), 91-122. MR 93h:32046

[14] I.I. Pyatetskii-shapiro, Automorphic Functions and the Geometry of Classical Domains, Gordon and Breach, New York, 1969.

[15] Y.T. Siu, The complex analyticity of harmonic maps and the strong rigidity of compact Kähler manifolds, Ann. of Math. 112(1980), 73-111. MR 81j:53061

[16] Y.T. Siu, Strong rigidity of compact quotients of exceptional bounded symmetric domains, Duke Math. J. 48(1981), 857-871. MR 86h:32053

[17] I-H. Tsai, Rigidity of holomorphic maps between compact Hermitian symmetric spaces, J. Diff. Geom. 33(1991), 717-729. MR 92d:32047

[18] I-H. Tsai, Rigidity of proper holomorphic maps between symmetric domains, J. Diff. Geom. 37(1993), 123-160. MR 93m:32038 
[19] Z.-H. Tu, Rigidity of proper holomorphic maps between bounded symmetric domains, Ph.D. Thesis, The University of Hong Kong, May 2000.

[20] A.E. Tumanov and G.M. Henkin, Local characterization of holomorphic automorphisms of classical domains, Dokl. Akad. Nauk SSSR 267(1982), 796-799. (Russian) MR 85b:32048

[21] J.A. Wolf, Fine structure of Hermitian symmetric spaces, in Geometry of Symmetric Spaces (Boothby-Weiss, eds), Marcel-Dekker, New York, 1972, 271-357. MR 53:8516

Department of Mathematics, The University of Hong Kong, Pokfulam Road, Hong KONG

E-mail address: Tuzhenhan@yahoo.com

Current address: Department of Mathematics, Huazhong University of Science and Technology, Wuhan, Hubei 430074, People's Republic of China 